\title{
Outcomes of children with psychiatric SLE
}

D espite the frequency with which neuropsychiatric manifestations affect children with systemic lupus erythematosus (SLE), little is known about how best to treat them, and less still about their long-term outcomes. Now, follow-up data from a study of immunosuppressive therapy in 53 children with neuropsychiatric SLE (NPSLE) provide encouraging evidence of sustained improvement. The paper, by Earl Silverman and colleagues and published in the Journal of Rheumatology, also sets out how future research could be standardized to enable larger-scale analyses of data.

A higher frequency of neurological manifestations, part of greater disease activity in general, is a feature that distinguishes paediatric SLE from adultonset disease. Nevertheless, NPSLE in children has rarely been studied in its own right. Indeed, the study by Silverman and coworkers is "one of the first paediatric SLE papers to assess psychiatric disease in a standardized fashion," says Eyal Muscal, a researcher in paediatric SLE who was not involved in the study. "Little is known about this topic in children," he continues, adding that "few studies have followed patients past the acute psychotic episode."

To address this deficit, Silverman and colleagues conducted a single-centre cohort study of children (aged $<18$ years at diagnosis) who had SLE according to the American College of Rheumatology classification criteria, psychiatric illness as a manifestation of active disease, and who were treated and followed between 1985 and July 2009. Patients with other psychiatric or reactive mood disorders were excluded. Psychiatric involvement, identified in the SLE clinic with screening questions, was confirmed after formal psychiatric evaluation. The collaboration with child psychiatrists was important, notes Muscal, who would nevertheless "have liked to see data from the formal cognitive testing or questionnaires used by psychiatrists to categorize cognitive defects."
The investigators report that all children diagnosed with psychiatric SLE had clinically relevant cognitive dysfunction, such as difficulties in concentration and memory that impaired performance at school, and that 75\% of them also had features of psychosis (mostly hallucinations). Thus, the researchers defined two groups: the cognitive dysfunction group $(n=13)$, and the psychosis plus cognitive dysfunction group $(n=40)$. Other disease features of SLE and autoantibody profiles were not significantly different between these two groups, nor so were the timing or duration of neurological manifestations.

All the children were treated with high-dose corticosteroids and a secondline immunosuppressive drug (except for two from the earliest period of the study who received only corticosteroids). Azathioprine was the second-line drug of choice $60 \%$ of the time, followed by intravenous cyclophosphamide (34\%) and mycophenolate mofetil (2\%). Response to therapy was evaluated every $2-6$ weeks until recovery, or until study end.

Key impediments to comparing results of previous small studies in childhood NPSLE have been the lack of definitions of response, and a neglect to report the use of concomitant psychiatric medications. The investigators thus predefined three possible outcomes (besides nonresponse): response, remission and relapse. Response and remission each required a 3-month period in which all psychiatric and cognitive symptoms were resolved without antipsychotic medication; response included a prednisone dose during that time $<50 \%$ of the maximum dose, and remission necessitated a dose $<10 \mathrm{mg}$ per day or $0.2 \mathrm{mg} / \mathrm{kg}$ per day, whichever was lower. Relapse applied to patients who experienced recurrence of symptoms requiring a $>50 \%$ increase in prednisone, antipsychotic medication and/or a switch in immunosuppressive therapy.

40 patients responded to therapy, and 25 fulfilled the criteria for remission. Of the 9

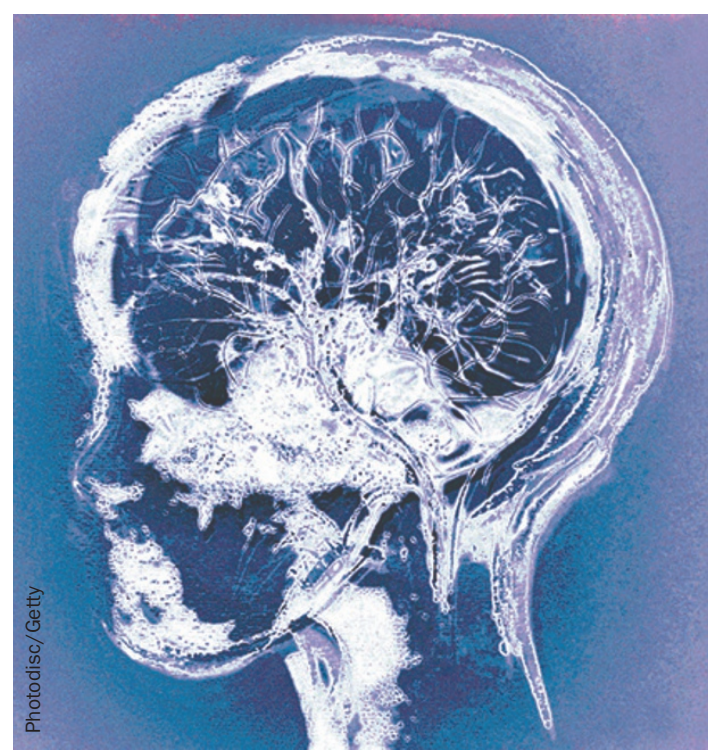

who did not respond, only 2 were followed for $>91$ weeks (the $95^{\text {th }}$ percentile of wholegroup time to response). Relapse occurred in 8 patients. These response rates were excellent, says Muscal, the rate of relapse was low and no children had chronic psychiatric illness. "Yet, time to complete remission was over 1 year for most of the children followed," he notes.

$56 \%$ of patients receiving azathioprine were switched to cyclophosphamide on account of poor response. Hence, the authors suggest that intravenous cyclophosphamide and high-dose prednisone be used as induction therapy. This recommendation might be firmed up as standardization of response measures "paves the way for multicentre cohort studies to be performed," says Muscal. Such efforts, it is hoped, will ultimately guide clinical care of children with NPSLE.

Emma Leah 